\title{
Generating functions for nth Collatz iteration
}

Benedict Irwin $^{1}$

${ }^{1}$ University of Cambridge

January 7, 2021

\section{Generating Function for $n^{\text {th }}$ Collatz Iteration}

We can consider the generating function for the Collatz map applied to the positive integers. Define

$$
C(n)= \begin{cases}n / 2 & n \bmod 2=0 \\ 3 n+1 & n \bmod 2=1\end{cases}
$$

and define the $m^{\text {th }}$ composition of the function as $C_{m}(n)$, such that $C_{0}=n$ and $C_{1}(n)=C(n)$ and $C_{2}(n)=C(C(n))$.

The generating function for positive integers is

$$
G_{0}(x)=\frac{x}{(1-x)^{2}}
$$

for the first iteration we have numbers $4,1,10,2,16,3, \ldots$

$$
G_{1}(x)=\frac{x}{\left(1-x^{2}\right)^{2}}\left(4+x+2 x^{2}\right)
$$

for the second iteration giving $2,4,5,1,8,10,11,2,14, \ldots$ we have

$$
G_{2}(x)=\frac{x}{\left(1-x^{4}\right)^{2}}\left(2+4 x+5 x^{2}+x^{3}+4 x^{4}+2 x^{5}+x^{6}\right)
$$

the next iteration is

$$
G_{3}(x)=\frac{P_{3}(x)}{\left(1-x^{8}\right)^{2}}
$$

in general this gives

$$
G_{n}(x)=\frac{P_{n}(x)}{\left(1-x^{2^{n}}\right)^{2}}
$$

for a polynomial of which seems to be order $2^{n+1}-1$ these polynomials appear to be related to the current iteration sequence by the following relationship

$$
P_{n}(x)=\left(\sum_{k=1}^{2^{n}} C_{n}(k) x^{k}\right)+\left(\sum_{k=2^{n}+1}^{2^{n+1}-1}\left(C_{n}(k)-2 C_{n}\left(k-2^{n}\right)\right) x^{k}\right)
$$

which we can write as

$$
P_{n}(x)=\left(\sum_{k=1}^{2^{n+1}-1} C_{n}(k) x^{k}\right)-2\left(\sum_{k=2^{n}+1}^{2^{n+1}-1} C_{n}\left(k-2^{n}\right) x^{k}\right)
$$


We could consider the Cauchy product of this and the simple series

$$
\frac{1}{\left(1-x^{2^{n}}\right)^{2}}=1+2 x^{2^{n}}+3 x^{2 \cdot 2^{n}}+4 x^{3 \cdot 2^{n}}+\cdots
$$

what does this mean? This means that for any level of iteration, we can describe the coefficient for any number, however large, using the first few function evaluations and a composition. However the expressions rapidly become complicated, with $2^{n+1}$ terms.

What conditions would then be required for a coefficient to be 1? For a given iteration this will depend on the number of ways to write a target number $t$, as the sum of an integer in the range $\left[1,2^{n}-1\right]$ and any of $\left[0,2^{n}, 2 \cdot 2^{n}, 3 \cdot 2^{n}, \cdots\right]$, for one iteration that's combinations in $[1,2,3]+[0,2,4,6,8, \cdots]$ which can make $[1,2,3],[3,4,5],[5,6,7]$ and so on indicating there are multiple ways to make $3,5,7, \cdots$.

All of the coefficient terms are positive which is nice. The only way a coefficient can be 1 in this iteration is if it is 1 in the polynomial, and multiplied by the 1 in the expanded series.

This means we can look at a subset of the polynomial, namely $P_{n}(x)$. We can then ask, how can a coefficient become 1 in $P_{n}(x)$ ? We can see that $C_{n}(k)>2 C_{n}\left(k-2^{n}\right)$ for $k \in\left[2^{n}+1,2^{n+1}-1\right]$ to keep the terms positive and non-zero. 